\title{
Efficacy of a trunk orthosis with joints providing resistive force on low-back load in elderly persons during static standing
}

\section{Junji Katsuhira' \\ Ko Matsudaira ${ }^{2}$ \\ Tadashi Yasui ${ }^{3}$ \\ Shinno lijima ${ }^{4}$ \\ Akihiro Ito ${ }^{4}$}

'Department of Nursing and

Rehabilitation Science at Odawara, International University of Health and Welfare, Odawara, Kanagawa, ${ }^{2}$ Department of Medical Research and Management for Musculoskeletal Pain, 22nd Century Medical and Research Center, The University of Tokyo, Tokyo, ${ }^{3}$ Kawamura-Gishi Company, Ltd., Daito-shi, Osaka, ${ }^{4}$ Graduate School of International University of Health and Welfare, Otawara, Tochigi, Japan

This article was published in the following Dove Press journal:

Clinical Interventions in Aging

I September 2015

Number of times this article has been viewed

Purpose: Postural alignment of elderly people becomes poor due to aging, possibly leading to low-back pain and spinal deformity. Although there are several interventions for treating these conditions, no previous study has reported the effectiveness of a spinal orthosis or lumbosacral orthosis (LSO) in healthy elderly people without specific spinal deformity. We therefore developed a trunk orthosis to decrease low-back muscle activity while training good postural alignment through resistive force provided by joints with springs (here, called the ORF, which stands for orthosis with joints providing resistive force) as a preventive method against abnormal posture and low-back pain in healthy elderly persons.

Patients and methods: Fifteen community-dwelling elderly men participated in this study. Participants stood freely for 10 seconds in a laboratory setting under three conditions: without an orthosis, with the ORF, and with an LSO. The Damen corset LSO was selected as it is frequently prescribed for patients with low-back pain. Postural alignment during static standing was recorded using a three-dimensional motion capture system employing infrared cameras. Two force plates were used to record center of pressure. Electromyograms were obtained for bilateral erector spinae (ES), left internal abdominal oblique, and right gluteus medius muscles.

Results: Pelvis forward tilt angle tended to increase while wearing the ORF and decrease while wearing the LSO, but these results were not significant compared to no orthosis. Thorax extension angle and thorax angle on pelvis coordinate system significantly increased while wearing the ORF compared to the other two conditions. ES activity significantly decreased while wearing the ORF compared to the other two conditions. Internal oblique activity was significantly smaller while wearing the LSO than with no orthosis. Center of pressure did not significantly differ among the conditions.

Conclusion: The ORF significantly improved trunk alignment and decreased ES activity in healthy elderly subjects during static standing.

Keywords: muscular activity, center of pressure, standing posture, spine

\section{Introduction}

Postural alignment worsens gradually over the course of aging. ${ }^{1}$ This poor postural alignment, which manifests as spinal kyphosis, can result in irreversible degeneration

Correspondence: Junji Katsuhira Department of Nursing and Rehabilitation Science at Odawara, International University of Health and Welfare, 1-2-25 Shiroyama, Odawara,

Kanagawa 250-8588, Japan

$\mathrm{Tel}+8 \mid 465216704$

Fax +8I 465216745

Email katsuhir@iuhw.ac.jp of the intervertebral disks and ligaments. It also often causes postural instability and leads to vertebral bone fracture and increased risk of falling. ${ }^{2,3}$

Due to these problems, various interventions are used for elderly persons who have spinal deformity to improve postural alignment. Battaglia et $\mathrm{al}^{4}$ reported that exercise improved spinal flexibility, and Imagama et $\mathrm{al}^{5}$ reported the improvement of lumbar lordosis angle, sagittal balance, and back muscle strength in elderly patients 
through a training program that included muscle strength and spinal range of motion exercises.

Spinal orthoses provide a way to directly modify the posture of elderly persons with spinal misalignment. Piffer et $\mathrm{al}^{6}$ reported that use of a newly designed spinal orthosis, the Spinomed $^{\circledR}$ (medi GmbH \& Co. KG, Bayreuth, Germany), for 6 months improved muscle strength, body balance, kyphosis angle, and vital capacity in elderly patients with osteoporosis. Ishida et $\mathrm{al}^{7}$ reported that a rucksack-type orthosis instantly modified spine alignment and decreased erector spinae (ES) activity in elderly patients with kyphosis.

As mentioned earlier, previous studies have reported that exercise and orthotic therapy are effective in treating the elderly with spinal misalignment. It is, however, also important to offer preventive intervention for the healthy elderly. Costantino et $\mathrm{al}^{8}$ reported that chronic low-back pain (LBP) in elderly people without specific spinal deformity could be effectively treated using a back school program, including exercise therapy for rehabilitation. However, to our knowledge, no previous studies have reported the effectiveness of a spinal orthosis or lumbosacral orthosis (LSO) in healthy elderly people without specific spinal deformity. A review of data from the Cochrane Database also found no evidence for the efficacy of lumbar support for decreasing low-back load. ${ }^{9}$ In addition, Rostami et al ${ }^{10}$ reported that use of an LSO for 4 weeks resulted in decreased trunk core muscle volume, and therefore, the drawback of long-term corset use may exceed the benefits.

In a previous study, to provide a preventive method against abnormal posture and LBP in elderly persons, we designed a trunk orthosis to address these issues by training good postural alignment while decreasing low-back load via resistive force provided by joints with springs (Figure 1). ${ }^{11}$ This orthosis with joints providing resistive force (hereafter, the ORF) produces a resistive moment that rotates the trunk backward and the pelvis forward (Figure 2). Resistive moment applied to the trunk can not only rotate it backward to shift the center of gravity of the head, arms, and trunk to the L4/L5 joint but also directly decrease low-back extension moment because the resistive moment work is in the same direction as that produced by ES activity. In addition, reaction moment can promote forward rotation of the pelvis, and this effect can also facilitate extension of the thorax. The ORF may therefore improve alignment and decrease ES activity in elderly people. Indeed, our recent studies reported that the ORF improved trunk alignment and gait performance of hemiparetic patients ${ }^{11}$ and elderly persons during level walking. ${ }^{12}$ However, we did not examine the effect of the ORF on ES activity and spinal alignment during static standing.

In light of the finding of no decrease in low-back muscle activity using LSOs, ${ }^{9}$ this study aimed to examine, through
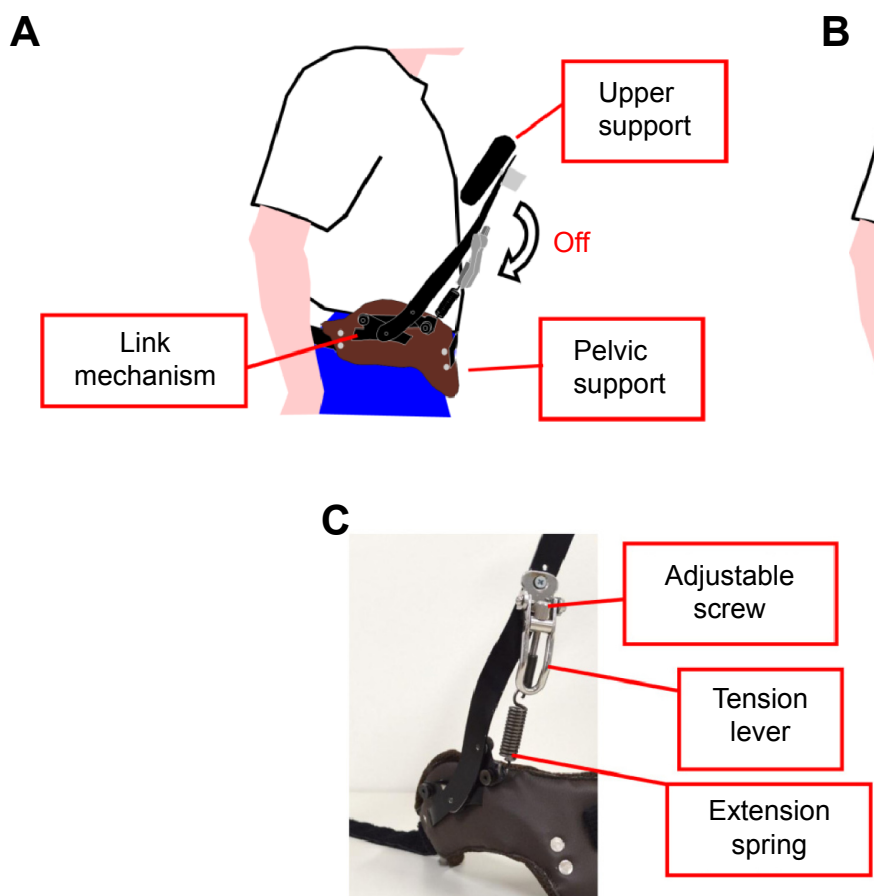

B

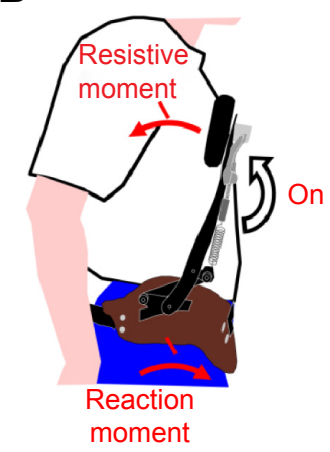

Figure I Trunk ORF.

Notes: (A) Wearing ORF without resistive force on the chest, (B) wearing ORF with resistive force on the chest, (C) detail of link mechanism. Abbreviation: ORF, orthosis with joints providing resistive force. 

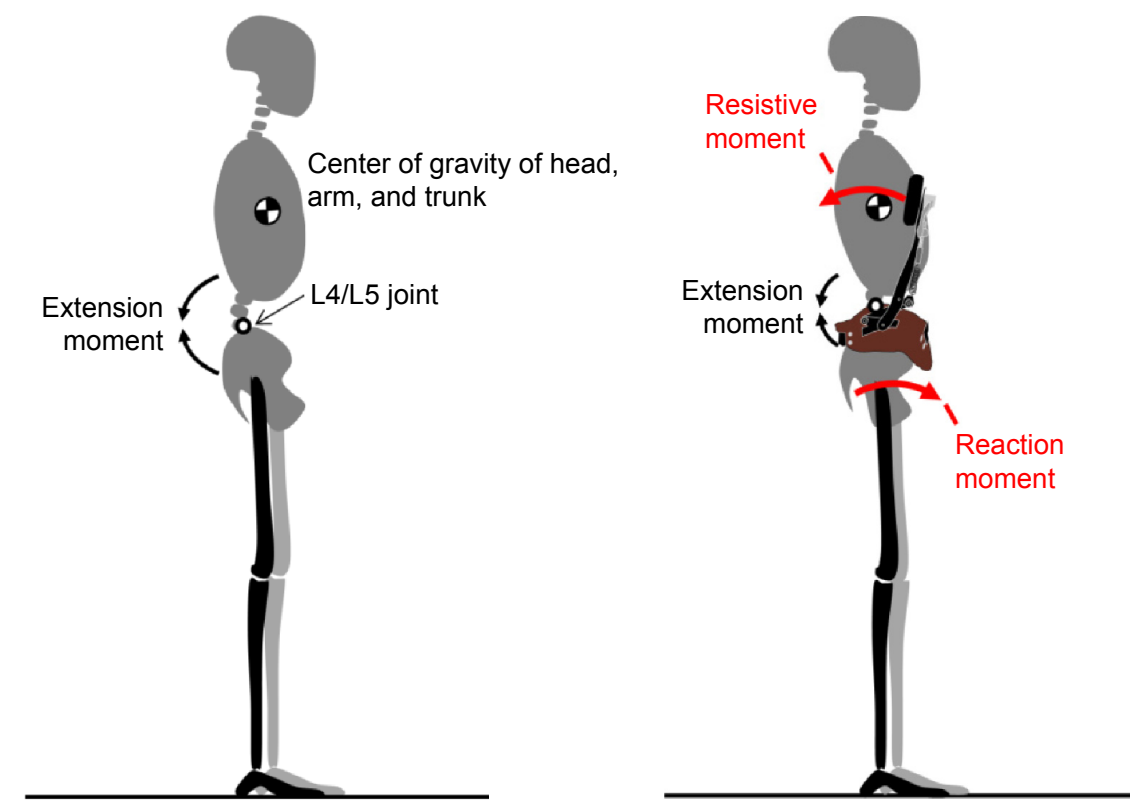

Figure 2 Biomechanical effect of ORF while static standing.

Abbreviation: ORF, orthosis with joints providing resistive force.

biomechanical analysis, the effects of the ORF on healthy elderly people during static standing and to compare the effects with those of an LSO and no orthosis. We hypothesized that wearing the ORF would effectively decrease ES activity and modify trunk alignment in healthy elderly people during static standing.

\section{Material and methods Participants}

We enrolled 15 healthy community-dwelling elderly men (mean age, 67.7 \pm 6.1 years; mean height, $162.4 \pm 5.7 \mathrm{~cm}$; mean weight, $62.3 \pm 7.8 \mathrm{~kg}$ ) from a group of 31 candidates. We excluded those with neurological disease, pain, history of orthopedic surgery, history of orthopedic treatment within the past 5 years, and history of LBP within the past 1 year. In the first phase of recruitment, we identified only two female subjects willing to participate in our study; however, due to a history of orthopedic treatment within the past 5 years, they were excluded. Therefore, we decided not to include any female subjects to mitigate the potential effects of sex and orthopedic disease. All participants provided written informed consent, and the study was approved by the ethics committees of the participating institutions, International University of Health and Welfare.

\section{Features of the ORF}

The features of the ORF (Figures 1 and 2) were described in our previous report. ${ }^{11}$ Briefly, the ORF weighs $0.99 \mathrm{~kg}$ and has a $40^{\circ}$ range of motion. Pelvic and upper supports are placed on the ileum and sternum, respectively. Stainless steel joints are connected to the upper support, with a nylon pad, and also to the pelvic support. These joints employ extension springs to produce tension, which is translated by a link mechanism into a resistive moment on the chest and a reaction moment on the posterior pelvis. The upper support initially inclines backward to exert resistive force on the chest and is then released via a mechanism that pulls tension levers downward. The resistive force can be increased or decreased via adjustment screws. The ORF is currently an investigational product that is not FDA-approved or approved by the corresponding national agency for the indication described herein.

\section{Experimental conditions}

Participants freely stood for 10 seconds under three conditions in the laboratory setting: with no orthosis, with the ORF, and with an LSO (Damen Corset, Pacific Supply, Osaka, Japan; Figure 3). As the Damen corset is frequently prescribed for patients with LBP, it was selected for use in this study. Our previous study reported carry-over effects of the ORF on body alignment of hemiparetic patients after removal while level walking. ${ }^{11}$ We therefore decided to measure two trials without intervention (no orthosis) first, after which subjects completed the two orthosis trials in random order. Participants were given 5 minutes to become accustomed to wearing the orthoses, and there was a minimum rest interval of 5 minutes between conditions. Resistive force exerted on the chest was 

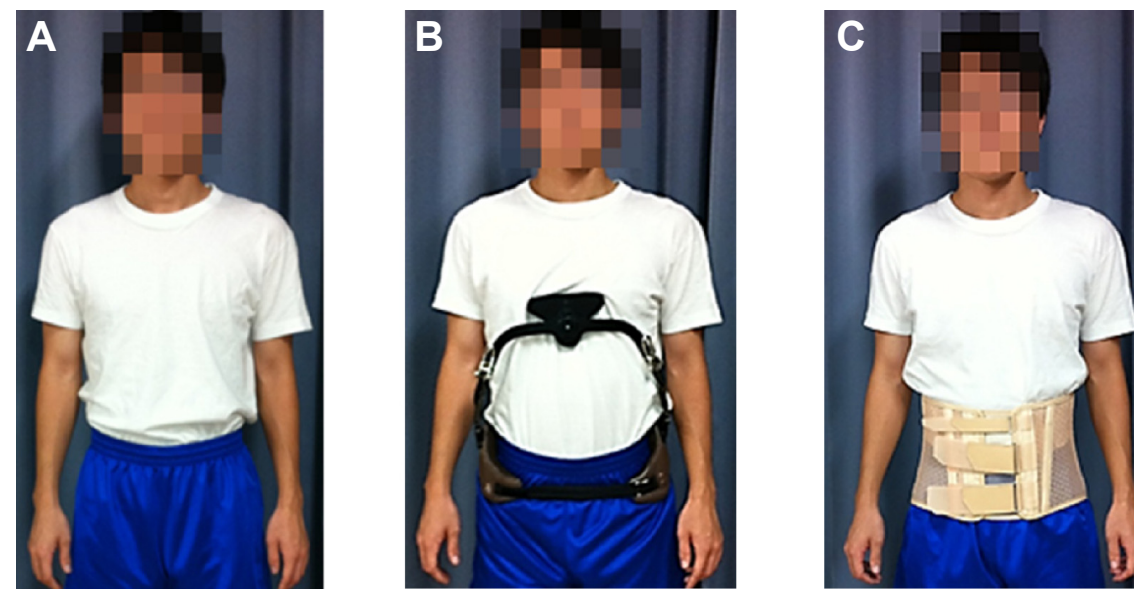

Figure 3 Experimental conditions.

Notes: (A) Without an orthosis, (B) with the LSO, (C) with the ORF.

Abbreviations: ORF, orthosis with joints providing resistive force; LSO, lumbosacral orthosis.

measured in real time with a strain gauge (Compression load cell LCN-A, Kyowa, Tokyo, Japan). The force data were transferred to a laptop via Bluetooth. Force was set to 20-25 $\mathrm{N}$ during static standing, and pressure between the corset and abdomen was set to $10 \mathrm{mmHg}$ in all measurement conditions. ${ }^{13}$ To obtain maximal voluntary contraction (MVC) values for normalization of individual muscle activities, participants performed maximal isometric contractions against gravity, while the experimenter (a licensed therapist) applied maximum resistance. ${ }^{14}$ After practicing stable isometric contraction efforts, single maximal contractions of each muscle were recorded according to Daniels and Worthingham's Muscle Testing. Subjects performed contractions against gravity, with maximum resistance applied by the examiner in the supine position to obtain MVC of the left internal abdominal oblique (IO) muscle (lifting head and shoulders from the table with right elbow toward left knee against imposed resistance to the right shoulder region), in the prone position to obtain MVC of the bilateral ES (back extension with hands resting on head against imposed resistance to the scapular region), and in the side-lying position with test leg elevated to obtain MVC of the right GM muscle (abduction with limb slightly extended beyond the midline and the pelvis rotated slightly forward while imposing resistance to the lateral surface of the knee).

\section{Experimental setup}

Static standing was recorded with a three-dimensional (3D) motion capture system (Vicon 612, Vicon, Oxford, UK) that employed two force plates (AMTI, Watertown, MA, USA), 12 infrared cameras (sampling rate, $120 \mathrm{~Hz}$ ), and 13 infrared-reflective markers (diameter, $14 \mathrm{~mm}$ ) attached to the $\mathrm{C} 7$ spinous process, T12 spinous process, L5 spinous process, manubrium sterni, second sacral vertebra and bilateral acromion process, bilateral anterior and posterior superior iliac spine, and bilateral iliac crest (Figure 4). All markers were captured in a reference static standing position, and then, the bilateral anterior superior iliac spine and iliac crest markers were removed before initiating measurements because they interfered with wearing of the orthoses. The positions of these removed markers were then interpolated using the reference static trial. To measure low-back muscle activity during static standing, electromyograms employing active electrodes to decrease noise (Biometrics, Newport, UK) were obtained for bilateral ES ( $2 \mathrm{~cm}$ to the side between L4 and L5 vertebrae), ${ }^{15}$ left IO ( $2 \mathrm{~cm}$ below the anterior superior iliac spine [ASIS] aligned approximately $6^{\circ}$ from the line between bilateral ASIS), ${ }^{16}$ and right GM (2.5 cm below the line between the iliac crest and greater trochanter) ${ }^{17}$ (Figure 3 ). The target muscle to confirm the effect of the ORF orthosis was the bilateral ES. The IO is classified as a core abdominal muscle, and Rostami et al ${ }^{10}$ reported that IO volume decreased after long-term use of a corset. Also, hip abduction moment increased when elderly subjects wore the ORF while level walking in our previous study. ${ }^{12}$ Thus, unilateral IO and GM activities were included as supplementary measures in this study.

Electromyography (EMG) signals were measured at $1,080 \mathrm{~Hz}$ because the acquisition frequency should be a whole-number multiple of the sampling frequency of the Vicon system (Vicon) (120 Hz).

\section{Data analysis}

All signals, including marker displacements and analog EMG signals, were acquired by the Vicon Datastation (Vicon) and then synchronized by correcting for the difference in 

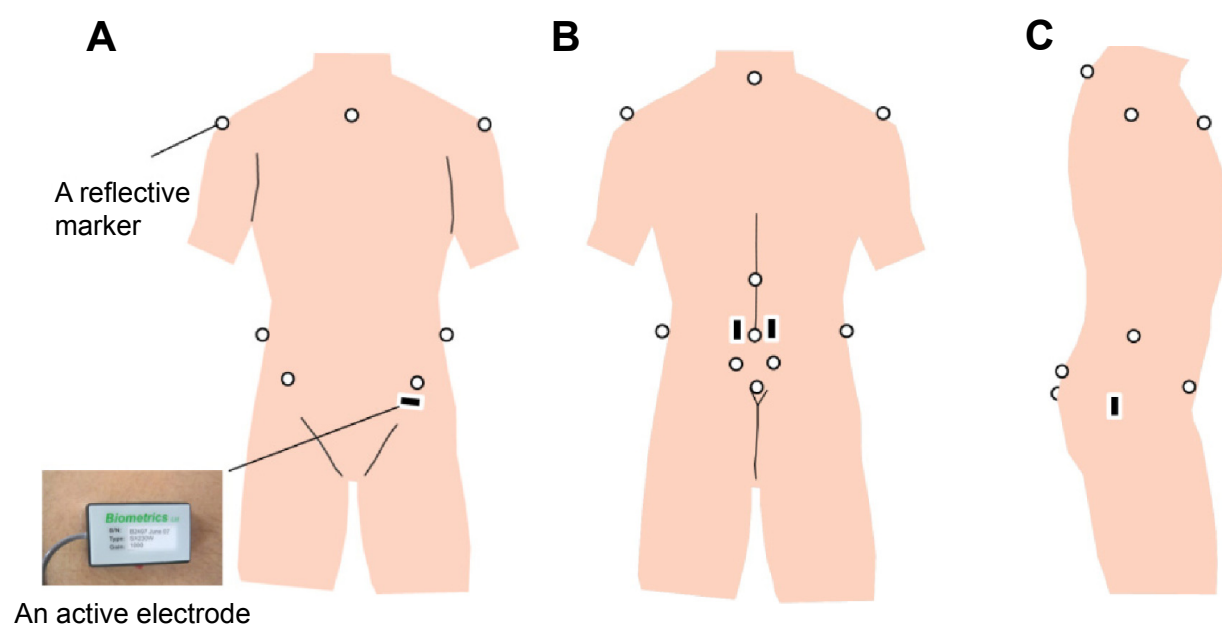

Figure 4 Positions of reflective markers and electrodes for electromyogram recording. Notes: (A) Anterior surface, $(\mathbf{B})$ posterior surface, $(\mathbf{C})$ lateral surface.

sampling frequency (120 vs 1,080 Hz) using Vicon Workstation software (Vicon). Band pass filter (20-420 Hz) was used during acquisition to decrease noise, ${ }^{18}$ and the data were then imported into Visual 3D analytical software (C-motion, Inc., Germantown, MD, USA) for kinetic and kinematic data analysis. Electromyograms were normalized to individual MVCs during isometric contraction (\%MVC). Root mean squares (RMS) for a $50 \mathrm{~ms}$ window were calculated, and integral values for these muscles (IEMG) were calculated. Consequently, Equation 1 is given as follows:

$$
\mathrm{IEMG}=\int_{0}^{t}\left[\mathrm{EMG}_{\mathrm{RMS}}\right](t) \mathrm{d} t
$$

where $\mathrm{EMG}_{\mathrm{RMS}}$ denotes $\mathrm{EMG}$ after conducting RMS for a $50 \mathrm{~ms}$ window and $t$ denotes 10 seconds, indicating the end time of static standing.

The physical coordinates and ground reaction force data were low-pass filtered with a second-order recursive Butterworth filter (cutoff frequencies 6 and $18 \mathrm{~Hz}$, respectively) according to Winter's technique. ${ }^{19}$ Also, the link segment model consisted of a trunk segment and a pelvis segment, and markers on each segment were used to calculate $3 \mathrm{D}$ trunk and pelvic angles using coordinate systems and the Eulerian method.

Center of pressure (COP) displacements are commonly used to evaluate balance performance in the elderly, ${ }^{20}$ and COP path length was validated as a performance outcome measure in a large group study. ${ }^{21}$ We therefore calculated COP path length to evaluate balance in the elderly subjects. The COP of the force vector of bilateral feet and COP total trajectory length were calculated for 10 seconds of standing.

\section{Statistical analysis}

Average kinetic, kinematic, and EMG values, as well as IEMG were acquired for 10 seconds of static standing. Mean average values of $3 \mathrm{D}$ trunk and pelvic angles, average EMG, and IEMG were calculated from two trials, per condition for analysis. Variables were compared by repeated measures analysis of variance (RT-ANOVA) followed by multiple pair-wise comparisons to Bonferroni correction. Statistical analysis was performed with SPSS20 (SPSS Inc., Chicago, IL, USA). Statistical significance was set at $P<0.05$.

\section{Results}

Average pelvis forward tilt angle, thorax extension angle, and thorax angle on the pelvis coordinate system are shown in Table 1 for the three static standing conditions: no orthosis, ORF, and LSO. One-way RT-ANOVA revealed a significant main effect of condition for all three angles. Pelvis forward tilt angle tended to increase while wearing the ORF and decrease while wearing the LSO, but these results were not significant compared to those without an orthosis. Thorax extension angle and thorax angle on the pelvis coordinate system were significantly increased while wearing the ORF compared to the other two conditions.

Integral and average muscular activities are shown in Table 2. One-way RT-ANOVA indicated significant main effect of condition on integral and average muscular activities of the bilateral ES and left IO, but not on the GM. Integral and average muscular activities of the bilateral ES were significantly smaller while wearing the ORF compared to no orthosis. For the left ES, integral and average muscular activities were significantly less while wearing the ORF compared to the LSO. The same trend was seen for 
Table I Mean and standard deviation for kinematic parameters in three standing conditions $(\mathrm{N}=15)$ and results of statistical analysis

\begin{tabular}{|c|c|c|c|c|c|c|c|}
\hline & $\begin{array}{l}\text { Without } \\
\text { ORF }\end{array}$ & $\begin{array}{l}\text { ORF mean } \\
\text { (SD) }\end{array}$ & LSO & $P$-value & $\begin{array}{l}\text { Without } \\
\text { ORF, ORF }\end{array}$ & $\begin{array}{l}\text { Without } \\
\text { ORF, LSO }\end{array}$ & $\begin{array}{l}\text { ORF, } \\
\text { LSO }\end{array}$ \\
\hline Pelvis forward tilt angle $\left({ }^{\circ}\right)$ & $6.13(5.07)$ & $8.13(6.55)$ & $4.46(5.77)$ & 0.010 & 0.281 & 0.165 & 0.050 \\
\hline Thorax extension angle $\left({ }^{\circ}\right)$ & $4.78(3.66)$ & $6.56(4.12)$ & $5.37(3.52)$ & $<\mathbf{0 . 0 0 1}$ & 0.004 & 0.172 & 0.021 \\
\hline $\begin{array}{l}\text { Thorax angle on pelvis } \\
\text { coordinate system: } \\
\text { extension }+\left(^{\circ}\right)\end{array}$ & $-2.40(7.02)$ & I.38 (7.62) & $-3.49(7.06)$ & 0.001 & 0.022 & 0.619 & 0.013 \\
\hline
\end{tabular}

Note: Data in bold are statistically significant, statistical significance was set at $P<0.05$.

Abbreviations: LSO, lumbosacral orthosis; ORF, orthosis with joints providing resistive force; SD, standard deviation.

the right ES, but it was not significant. Integral and average muscular activities of the left IO were significantly dimished while wearing the LSO compared to no orthosis, but there was no significant difference between ORF and no orthosis conditions.

COP trajectory is shown in Table 3 . No significant difference was observed among the three conditions.

\section{Discussion}

Incidence of LBP increases with age. ${ }^{22}$ Age-related spinal deformity as well as ES hyperactivity are among the major causes of LBP. ${ }^{18}$ We previously developed the ORF to modify trunk and pelvis alignment and decrease ES activity. We examined the effects of this orthosis on healthy elderly participants and found that the ORF could effectively modify trunk alignment while decreasing ES activity compared to an LSO or no orthosis. In fact, no significant positive effects were observed while wearing the LSO.

Coskun Benlidayi and Basaran ${ }^{23}$ reported that lumbar lordosis is significantly smaller in elderly than in young subjects. Wearing of the ORF in this study significantly extended the upper trunk and tended to tilt the pelvis forward, which would contribute to increasing lumbar lordosis. Lee et $\mathrm{al}^{13}$ reported the effectiveness of a lumbar belt and pelvic belt to modify pelvic and spinal alignment on posture in healthy young participants. Additionally, Piffer et $\mathrm{al}^{6}$ reported that use of the newly designed Spinomed ${ }^{\circledR}$ (medi GmbH \& Co. KG) orthosis for 6 months improved kyphosis in elderly patients with osteoporosis. However, the present study appears to be the first to report on an orthosis that can modify alignment in healthy elderly participants without spinal deformity or chronic LBP.

Most spinal orthoses were developed to treat LBP and abnormal spinal alignment. Based on the findings of this study, the ORF shows promise for the modification of spinal alignment in elderly people without any specific deformity or syndromes related to LBP. Previous studies reported that interventions teaching awareness of posture, such as lessons on the Alexander technique, could be beneficial in treating LBP, as well as cost-effective. ${ }^{24,25}$ Back school programs that include exercise therapy could also be effective in elderly people with chronic LBP with no specific spinal deformities. ${ }^{8}$ Therefore, the ORF's ability to modify trunk alignment in elderly people might be useful to increase awareness of

Table 2 Mean and standard deviation for muscular activities in three standing conditions $(\mathrm{N}=15)$ and results of statistical analysis

\begin{tabular}{|c|c|c|c|c|c|c|c|}
\hline $\begin{array}{l}\text { Muscle } \\
\text { activity }\end{array}$ & $\begin{array}{l}\text { Without } \\
\text { ORF }\end{array}$ & $\begin{array}{l}\text { ORF mean } \\
\text { (SD) }\end{array}$ & LSO & $P$-value & $\begin{array}{l}\text { Without } \\
\text { ORF, ORF }\end{array}$ & $\begin{array}{l}\text { Without } \\
\text { ORF, LSO }\end{array}$ & $\begin{array}{l}\text { ORF, } \\
\text { LSO }\end{array}$ \\
\hline \multicolumn{8}{|c|}{ Percent IEMG } \\
\hline Right ES & 57.75 (43.97) & 45.48 (32.77) & $53.58(38.77)$ & 0.003 & 0.024 & 0.433 & 0.055 \\
\hline Left ES & $81.27(51.07)$ & $60.81(51.15)$ & 78.87 (50.29) & $<0.001$ & 0.001 & 1.000 & 0.004 \\
\hline Right GM & 59.17 (53.07) & $53.30(60.42)$ & 67.14 (79.43) & 0.132 & & & \\
\hline Left IO & I52.23 (95.66) & I 32.09 (91.53) & I 27.77 (82.55) & 0.010 & 0.117 & 0.037 & 1.000 \\
\hline \multicolumn{8}{|c|}{ Percent MVC } \\
\hline Right ES & $5.86(4.53)$ & $4.60(3.33)$ & $5.43(3.96)$ & 0.003 & 0.027 & 0.435 & 0.053 \\
\hline Left ES & $8.17(5.09)$ & $6.10(5.10)$ & $7.92(5.01)$ & $<0.001$ & 0.001 & 1.000 & 0.004 \\
\hline Right GM & $5.92(5.30)$ & $5.33(6.40)$ & $6.72(7.94)$ & 0.132 & & & \\
\hline Left IO & I5.27 (9.52) & $13.25(9.12)$ & $12.83(8.22)$ & 0.009 & 0.114 & 0.037 & 1.000 \\
\hline
\end{tabular}

Notes: \%IEMG, integral of the EMG over 10 seconds relative to maximum. \%MVC, average EMG over 10 seconds relative to maximum. Data in bold are statistically significant, statistical significance was set at $P<0.05$.

Abbreviations: ES, erector spinae; GM, gluteus media; IEMG, integral electromyography; IO, internal oblique; MVC, maximum voluntary contraction; LSO, lumbosacral orthosis; ORF, orthosis with joints providing resistive force; SD, standard deviation. 
Table 3 Mean and standard deviation for COP trajectory in three standing conditions $(\mathrm{N}=15)$ and results of statistical analysis

\begin{tabular}{lllll}
\hline & Without ORF & ORF mean (SD) & LSO & P-value \\
\hline Trajectory of COP $(\mathrm{m})$ & $0.1285(0.0610)$ & $0.1314(0.0454)$ & $0.1304(0.0481)$ & 0.924 \\
\hline A
\end{tabular}

Abbreviations: COP, center of pressure; LSO, lumbosacral orthosis; ORF, orthosis with joints providing resistive force; SD, standard deviation.

proper posture and help prevent misalignment and spinal deformity.

The most significant effect of wearing the ORF in this study was to decrease ES activity. A systematic review reported that wearing an LSO alone could not decrease low-back load. ${ }^{9}$ However, Cholewicki et al ${ }^{18}$ reported that wearing an LSO could decrease ES activity during a postural control task such as sitting on an unstable seat. In this study, ES activity decreased slightly by $1 \%-2 \%$ MVC while wearing the ORF. Despite this small decrease, a modeling study showed that adding a $32 \mathrm{~kg}$ mass to the trunk required an increase in trunk muscle cocontraction of approximately $1 \%-2 \%$ MVC above the level normally necessary to maintain a stable upright position of the spine around the neutral posture. ${ }^{26}$ Furthermore, previous studies have reported that maintaining muscular contraction above $5 \%$ MVC may cause back-muscle fatigue and pain. ${ }^{27}$ In the present study, average right ES activity was below $5 \%$ MVC while wearing the ORF, but was higher for the other two conditions. In addition, average left ES activity decreased within the $6 \% \mathrm{MVC}$ range while wearing the ORF, but was approximately $8 \%$ for the other two conditions. These results indicate that the ORF was effective in decreasing the activity of low-back muscles, which was not observed while wearing the LSO. In a postural control task, trunk muscle activity does not usually exceed 3\% $\mathrm{MVC}$ in young participants. ${ }^{27}$ However, muscular activity of elderly people during MVC is lower, and therefore greater relative effort would be needed to maintain an upright standing position. Rostami et $\mathrm{al}^{10}$ reported that wearing an LSO led to decreased muscle volume in the abdominal side muscles, including the IO. Wearing the ORF in the present study did not significantly decrease IO activity, whereas wearing the LSO significantly decreased it, compared to no orthosis. These results support those of Rostami et al ${ }^{10}$ and suggest that resistive force might not decrease low-back muscle activity without a decrease in side abdominal muscle volume.

No significant changes in COP trajectory were observed while wearing either orthosis in this study. Cholewicki et a ${ }^{18}$ reported that COP displacement during an unstable sitting task did not significantly differ from the control condition (no orthosis) while wearing an LSO. Furthermore, Chen et $\mathrm{al}^{28}$ reported that their insole served to improve the stability index, as calculated using COP displacement in elderly participants. COP is mainly controlled by the ankle plantar flexors, and therefore intervention using foot-based orthotic devices may be more useful than trunk-based devices.

Wearing the ORF during static standing served to decrease ES activity and modify trunk alignment, which may be effective for the prevention and treatment of LBP and spinal deformity in elderly people. However, this study has several limitations. First, we did not confirm the effects of long-term ORF use, as wearing the ORF or LSO for lengthy periods might adversely affect muscle control. Second, participants were healthy elderly men only and this was a within-subject trial. Healthy elderly women and participants with LBP or low-back disorders should be included in future studies, including randomized controlled trials. Third, we confirmed reduction of ES activity, but it might not be the exact cause. Resistive moment generated by the ORF joints may have decreased extension moment exerted by the ES muscles, or greater thorax extension angle could have decreased ES activity by reducing the lever arm from the L4/L5 joint to gravitational force on the center of gravity of the upper trunk. Moreover, ES is a surface muscle. In a future study, we must confirm these changes in spinal loading using a more detailed biomechanical model including the deep back muscles, such as bilateral multifidus muscle.

\section{Conclusion}

This study revealed that the ORF significantly improved trunk alignment and decreased ES activity in healthy elderly participants. These findings suggest that the ORF may help prevent LBP and spinal deformity in elderly people. Further studies are needed to examine the use of the ORF in patients with LBP and spine deformity.

\section{Acknowledgment}

This study was supported by the dissemination project of Clinical Research for Occupational Injuries and Illness from Ministry of Health, Labour and Welfare of Japan. 


\section{Author contributions}

Provided substantial contribution to conception and design: JK, KM, TY. Involved with data collection and analysis: JK, SI, AI. Involved with interpretation of data: JK, SI, AI. Drafted the article: JK, KM. Revised the manuscript critically for important intellectual content: JK, KM, TY, SI, AI. Provided approval of the version to be published: JK, KM, TY, SI, AI.

\section{Disclosure}

The authors report no conflicts of interest in this work.

\section{References}

1. Gelb DE, Lenke LG, Bridwell KH, Blanke K, McEnery KW. An analysis of sagittal spinal alignment in 100 asymptomatic middle and older aged volunteers. Spine. 1995;20:1351-1358.

2. Nguyen T, Sambrook P, Kelly P, et al. Prediction of osteoporotic fractures by postural instability and bone density. BMJ. 1993;307:1111-1115.

3. Kasukawa Y, Miyakoshi N, Hongo M, et al. Relationships between falls, spinal curvature, spinal mobility and back extensor strength in elderly people. J Bone Miner Metab. 2010;28:82-87.

4. Battaglia G, Bellafiore M, Caramazza G, Paoli A, Bianco A, Palma A. Changes in spinal range of motion after a flexibility training program in elderly women. Clin Interv Aging. 2014;9:653-660.

5. Imagama S, Matsuyama Y, Hasegawa Y, et al. Back muscle strength and spinal mobility are predictors of quality of life in middle-aged and elderly males. Eur Spine J. 2011;20:954-961.

6. Pfeifer M, Kohlwey L, Begerow B, Minne HW. Effects of two newly developed spinal orthoses on trunk muscle strength, posture, and quality-of-life in women with postmenopausal osteoporosis: a randomized trial. Am J Phys Med Rehabil. 2011;90:805-815

7. Ishida H, Watanabe S, Yanagawa H, Kawasaki M, Kobayashi Y, Amano Y. Immediate effects of a rucksack type orthosis on the elderly with decreased lumbar lordosis during standing and walking Electromyogr Clin Neurophysiol. 2008;48:53-61.

8. Costantino C, Romiti D. Effectiveness of Back School program versus hydrotherapy in elderly patients with chronic non-specific low back pain: a randomized clinical trial. Acta Biomed. 2014;85:52-61.

9. van Duijvenbode I, Jellema P, van Poppel M, van Tulder MW. Lumbar supports for prevention and treatment of low back pain. Cochrane Database Syst Rev. 2008:Cd001823.

10. Rostami M, Noormohammadpour P, Sadeghian AH, Mansournia MA, Kordi R. The effect of lumbar support on the ultrasound measurements of trunk muscles: a single-blinded randomized controlled trial. PMR. 2014;6:302-308; quiz 308.

11. Katsuhira J, Miura N, Yasui T, Takane M, Sumiko Y. Efficacy of a newly designed trunk orthosis with joints providing resistive force in adults with post-stroke hemiparesis. Prosthet Orthot Int. Epub August 18, 2014.
12. Iijima S, Katsuhira J, Ito A, Nomura T, Maruyama H. Effects of a trunk brace with joints that provides a resistive force to modify pelvic alignment during level walking in the elderly. Phys Ther Japan. 2014;41: 355-363.

13. Lee ES, Ko CW, Suh SW, Kumar S, Kang IK, Yang JH. The effect of age on sagittal plane profile of the lumbar spine according to standing, supine, and various sitting positions. J Orthop Surg Res. 2014;9:11.

14. Montgomery J, Hislop H, Connelly B. Daniels and Worthingham's Muscle Testing: Techniques of Manual Examination. 8th ed. Maryland Heights, MO: Saunders/Elsevier; 2007.

15. De Foa JL, Forrest W, Biedermann H. Muscle fibre direction of longissimus, iliocostalis and multifidus: landmark-derived reference lines. J Anat. 1989;163:243.

16. Ng JK, Kippers V, Richardson CA. Muscle fibre orientation of abdominal muscles and suggested surface EMG electrode positions. Electromyogr Clin Neurophysiol. 1998;38:51-58.

17. Perotto A, Delagi EF, Iazzetti J, Morrison D. Anatomical Guide for The Electromyographer: The Limbs and Trunk. Springfield, IL: Charles C Thomas; 2005.

18. Cholewicki J, Reeves NP, Everding VQ, Morrisette DC. Lumbosacral orthoses reduce trunk muscle activity in a postural control task. J Biomech. 2007;40:1731-1736.

19. Winter DA. Biomechanics and Motor Control of Human Movement. Hoboken, NJ: John Wiley \& Sons; 2009.

20. Kalisch T, Kattenstroth JC, Noth S, Tegenthoff M, Dinse HR. Rapid assessment of age-related differences in standing balance. J Aging Res. 2011;2011:160490

21. Donath L, Roth R, Zahner L, Faude O. Testing single and double limb standing balance performance: comparison of COP path length evaluation between two devices. Gait Posture. 2012;36:439-443.

22. Dunn KM, Hestbaek L, Cassidy JD. Low back pain across the life course. Best Pract Res Clin Rheumatol. 2013;27:591-600.

23. Coskun Benlidayi I, Basaran S. Comparative study of lumbosacral alignment in elderly versus young adults: data on patients with low back pain. Aging Clin Exp Res. 2015;27:297-302.

24. Little P, Lewith G, Webley F, et al. Randomised controlled trial of Alexander technique lessons, exercise, and massage (ATEAM) for chronic and recurrent back pain. BMJ. 2008;337:a884

25. Hollinghurst S, Sharp D, Ballard K, et al. Randomised controlled trial of Alexander technique lessons, exercise, and massage (ATEAM) for chronic and recurrent back pain: economic evaluation. BMJ. 2008;337:a2656.

26. Cholewicki J, Panjabi MM, Khachatryan A. Stabilizing function of trunk flexor-extensor muscles around a neutral spine posture. Spine. 1997; 22:2207-2212.

27. Bjorksten M, Jonsson B. Endurance limit of force in long-term intermittent static contractions. Scand J Work Environ Health. 1977;3:23-27.

28. Chen TH, Chou LW, Tsai MW, Lo MJ, Kao MJ. Effectiveness of a heel cup with an arch support insole on the standing balance of the elderly. Clin Interv Aging. 2014;9:351-356.
Clinical Interventions in Aging

\section{Publish your work in this journal}

Clinical Interventions in Aging is an international, peer-reviewed journal focusing on evidence-based reports on the value or lack thereof of treatments intended to prevent or delay the onset of maladaptive correlates of aging in human beings. This journal is indexed on PubMed Central, MedLine,
Dovepress

CAS, Scopus and the Elsevier Bibliographic databases. The manuscript management system is completely online and includes a very quick and fair peer-review system, which is all easy to use. Visit http://www.dovepress. com/testimonials.php to read real quotes from published authors. 